\title{
BASIC MODELING FOR ELECTRIC TRACTION SYSTEMS UNDER UNCERTAINTY
}

L. Abrahamsson ${ }^{(1)}$ and L. Söder ${ }^{(1)}$

(1) KTH, Sweden \begin{abstract}
sensitivity, which is of our immediate interest. uncertainties.

\section{INTRODUCTION}

For about more than one decade, the railway has in many countries experienced a renaissance after several decades of decay, cut downs, and lack of new investments. The main reasons for the expansion of the railway are: environmental, economical, as well as related to safety issues. This in turn, has increased the interest in the development of models of the railway net.
\end{abstract}

ABSTRACT

The objective of this paper is initially to present a basic modeling of the railway traction system. This model includes the basic technologies used today. The voltage dependencies of the maximal possible power consumption as well as the maximal velocity of the common Rc-locomotives are included. The latter is very crucial for the studies of time table

Moreover, a method is presented, that estimates the expected train delay time for a given feeding technology. The reference timetable assumes the same train and surrounding conditions, but no voltage drops. In the numerical example where the developed model is applied to a realistic test system, a set of possible amounts of railway traffic are treated as

Mainly, the contributions of this paper are three: compiling and connecting already accepted models, the development of a method for numerical calculations using this model compilation, and an example to apply this model on.

When making a decision about the future costs, possible under investments or over investments need to be estimated in an appropriate manner. In a railway system, a voltage drop causes the trains to run slower, while in the ordinary power system some consumers occasionally have to be disconnected. Furthermore, short-time interruptions are nearly not noticed, thanks to the great momentum of a train set at higher speed.

One important issue is the consequence of under dimensioning. The objective of this paper is first to present a basic simulation method to estimate this, combining and using some accepted railway traction models. For a given choice of electric feeding technology, this simulation method determines the delay time of a train for a specified traffic situation, when the electric system is not infinitely strong. Here, a "delay time" is defined as the difference between the time it takes to travel a certain distance at nominal voltage level all the time and the simulated traveling time for the same distance. Secondly, the presented method will be applied to a specific case, in order to show the usability of the method.

The system in this study is exposed to different train traffic intensities - which can be considered as an uncertainty of the future.

\section{THE MODEL}

The Catenaries and the Transmission Lines

Two different catenary technologies are modeled, BT (Booster Transformer) and AT (Auto Transformer). These systems have their names from the kind of transformers that are used in each respective technological solution. One type of high voltage transmission lines (from here and on denoted HV lines) are also modeled. An example of how this HV line might be connected to the feeding system as illustrated in Figure 1. A common and handy approximation of BT catenaries as well as the HV lines is a $\Pi$-model impedance depending linearly on distance. The modeling of AT lines in [1] is basically the same as for BT and HV lines, however, a somewhat more detailed modeling of the AT-lines is given in [2]. In the latter which might suit better for this study, AT lines are separated into two different $\Pi$-model sections. One initial impedance in each feeding point, and one length-dependent impedance along the railway tracks.

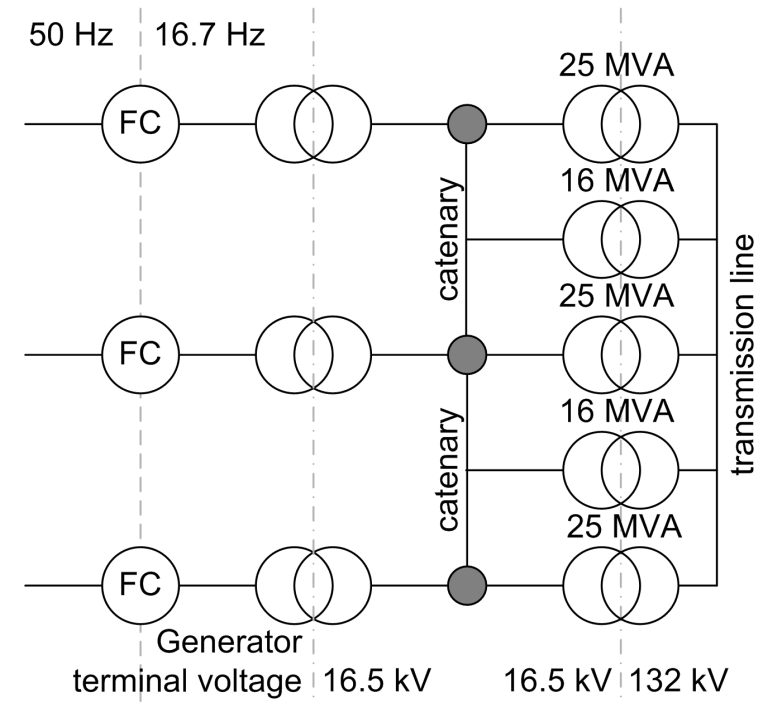

Figure 1 An illustration of the general idea of transformer usage

The Rc Locomotives

In this study, the in Sweden most common locomotives, the Rc-locomotives, are treated. Another reason to study the Rc 
trains - besides the major usage - is their peculiar (reactive) power consumption properties.

The model of the reactive power consumption of the Rc locomotives used is a simplification of the one found in [5], [6], and [7]. In that model the Rc trains are modeled as two nodes. One node connected to the catenary, and another node where the thyristor bridges rectifying the current to the dc motor are connected. The transformer between these nodes is in this paper modeled as an ideal impedance allowing a one node model.

Apart from the active power consumption, in the used model, the reactive power consumption of an Rc-locomotive also depends on the catenary ac voltage and the voltage of the $\mathrm{dc}$ motor of the train.

The dc voltage, $U_{d i \alpha}$ in p.u., that controls the dc motors of the train is related to the velocity, $v$ in $\mathrm{km} / \mathrm{h}$, of the train as

$$
U_{d i \alpha}=\left(\min \left\{E_{\max }, \frac{E_{\max }}{v_{\text {base }}} v\right\}\right) \cdot \frac{U_{b 2}}{U_{b 3} \cdot U_{b}}
$$

where $E_{\max }$ is the maximal dc voltage over the dc motor, and $v_{\text {base }}$ is the base velocity of the trains. Typical values are: $E_{\max }=770 \mathrm{~V}$ and $v_{\text {base }}=78 \mathrm{~km} / \mathrm{h} \mathrm{[6],} \mathrm{[7].} \mathrm{The} \mathrm{base}$ voltage $U_{b}=16.5 \mathrm{kV}$, the nominal catenary voltage. The scalings $U_{b 2}=15 \mathrm{kV}$ and $U_{b 3}=487 \mathrm{~V}$ arises from the base voltages of the ideal transformer. The base voltage $U_{b}=16.5$ $\mathrm{kV}$, the nominal catenary voltage. Moreover, the dc motor current, $I_{D}$, obeys the relation

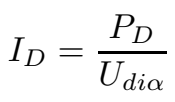

according to [5] and [6]. The reactive power demand of the locomotive is described as

$$
Q=\left\{\begin{array}{c}
U \cdot I_{D} \cdot \frac{\sqrt{2}}{\pi} \cdot \sin \left(\arccos \left(\frac{U_{d i \alpha} \cdot \pi}{\sqrt{2} \cdot U}-3\right)\right) \\
\text { when } \frac{U_{d i \alpha} \cdot \pi}{4 \cdot \sqrt{2}} \leq U \leq \frac{U_{d i \alpha} \cdot \pi}{2 \cdot \sqrt{2}} \\
U \cdot I_{D} \cdot \frac{\sqrt{2}}{\pi} \cdot \sin \left(\arccos \left(\frac{U_{d i \alpha} \cdot \pi}{\sqrt{2} \cdot U}-1\right)\right) \\
\text { when } U \geq \frac{U_{d i \alpha} \cdot \pi}{2 \cdot \sqrt{2}} .
\end{array}\right.
$$

\section{The Frequency Converters}

The active power production by a rotary frequency converter (FC in Figure 1) is determined by some machine parameters and the phase difference between the $50 \mathrm{~Hz}$ and the $16.7 \mathrm{~Hz}$ sides of the converter. The static frequency converters, can among other things control their active power production. When setting the static converters to mimic rotary converters, the modeling is quite similar to the rotating ones [6], [7].

The model used for the rotating converters originates from [5], [6], where the frequency converters are PU-like nodes with non-constant power. Moreover, it assumes the generator side voltage of the frequency converter, $U^{g}$ to be controlled at $16.5 \mathrm{kV}$. The relation between power production and the system voltages / angles can be concluded in the four equations

$$
\theta^{0}=\theta^{50}-\frac{1}{3} \cdot \arctan \frac{X_{50} \cdot P_{G}}{\left(U^{m}\right)^{2}-X_{50} \cdot Q_{50}},
$$

$$
\begin{gathered}
X_{q}^{m_{\text {new }}} \cdot P_{G}=\frac{X_{q}^{m} \cdot P_{G}}{\left(U^{m}\right)^{2}+X_{q}^{m} \cdot Q_{50}}, \\
\psi=-\frac{1}{3} \arctan X_{q}^{m_{\text {new }}} \cdot P_{G^{-}} \\
-\arctan \frac{X_{q}^{g} \cdot P_{G}}{\left(U^{g}\right)^{2}+X_{q}^{g} \cdot Q_{G}},
\end{gathered}
$$

and

$$
\begin{aligned}
f & =\theta-\theta^{0}-\psi\left(P_{G}, Q_{G}, U\right) \\
& =0
\end{aligned}
$$

where $Q_{50}$ denotes the reactive power production on the 50 $\mathrm{Hz}$ net (motor) side, $X_{50}$ is the reactance of the $50 \mathrm{~Hz}$ system, $\theta^{50}$ is the phase angle on the $50 \mathrm{~Hz}$ side, $X_{q}^{m}$ and $X_{q}^{g}$ are quadrature reactances of motor and generator respectively, $U^{m}$ and $U^{g}$ are the motor and generator side voltages, $P_{G}$ and $Q_{G}$ are the generated active and reactive powers at the generator side.

In real life there are often more than one converter at a converter station, and they are parallel connected. In this paper a simpler version of the reality is modeled. Here, there is no physical limitation of the possible apparent power a single converter can deliver, and thereby merely one converter is located at each converter station.

\section{THE METHOD}

\section{General}

The aim of the method developed is to simulate the train delays caused by voltage drops. To be specific, the purpose of the model is to compare the simulated time for a train to travel a certain distance under certain given circumstances to the time it would take for the train to travel the same distance with an infinitely strong feeding system. The method assumes pseudo-statical conditions in several ways. Electrically, a static model is used in order to evaluate the voltages, angles and power flows in each discretized segment of time. That problem is formulated as an ordinary system of equations to be solved numerically by the method of NewtonRaphson. In reality, of course the electric system is subject of dynamics, where the prior states might influence the subsequent ones. This influence is nevertheless assumed to be negligible in this study.

Physically, the proposed method works pseudo-statical as well. The maximal stationary velocities of the trains are determined by a voltage dependency. In determination of the maximal velocity, constant velocity is assumed. At the same time, the maximal velocity varies with voltage, and the voltage varies with train location and the interaction with the other trains. Consequently, this means that a change in catenary voltage level from one segment in time to another will change the velocity of the train immediately without any consideration of the train mass inertia. The usage of a stationary model for moving trains is not really justified by other means than the fact that avoiding dynamics makes life a lot easier. Note that the actual velocity of a train can be less than or equal to the maximal attainable one in general. However, in this study the trains are assumed to drive as fast as possible all the time.

Another simplification in the proposed method regards the tracks. With respect to the railway feeding, the method as- 
sumes single track railway, while, on the other hand mechanically double tracks are assumed. This simplification means that the trains are able to meet or pass each other everywhere, but still there is only one catenary line connecting every pair of cities.

\section{Rc-locomotives}

In order to be able to study delays caused by voltage drops, a model that somehow relates the greatest attainable velocity to the catenary voltage level is needed. This relation, stated in equation 8 , can be derived as follows: The maximal power consumption of an Rc locomotive is known as a function of constant train velocity for a few voltage levels. When the resistive power of a train set running at constant velocity under certain circumstances as a function of velocity is known, as well as the pantograph voltage level, the intersection point of the maximal power consumption curve and the resistive power curve can be determined. This point gives the maximal attainable constant velocity, as well as the corresponding maximal possible power consumption of the locomotive at the present voltage level.

In the literature, e.g. [4] and [9], force-velocity relations are commonly used. Since stationarity is assumed, the relation $P=v \cdot F$, where $v$ denotes velocity and $F$ denotes force can be used. That assumption will be used though there obviously must be accelerations and decelerations involved.

The resistive force function of a constant running velocity, can be found in [9] for a couple of train setups and ascents / descents. Power usage related to e.g. train heating and internal power are not included in the developed model.

Rc locomotives can have maximal speeds of either 135, 160 or $180 \mathrm{~km} / \mathrm{h}$ [4]. Since goods trains are assumed, $135 \mathrm{~km} / \mathrm{h}$ is suitable for this study. However, the method can handle all the three types.

In order to simplify things, the five intersection points seen from the right hand side of the example plot of Figure 4 are calculated once and for all, for all resistive-power curves that are used by the method. This is done in order to retrieve simple expressions for the voltage dependencies of the locomotive's maximal active power demand as well as the maximal velocity of the train. By least squares approximation, two affine relations are extracted:

$$
\begin{aligned}
P_{D} & =\frac{a \cdot U \cdot U_{b}+b}{S_{b}} \\
v & =c \cdot U \cdot U_{b}+d
\end{aligned}
$$

( $v$ in $\mathrm{km} / \mathrm{h}, P$ and $U$ in p.u.). This approximation suits well for most interesting situations. In order not to complicate things (especially not the partial derivatives of $Q_{D}$ ) any further, no limitation velocity $v_{\max }=135 \mathrm{~km} / \mathrm{h}$ was introduced. The model is valid only under "non-extreme" circumstances.

\section{THE METHOD ALGORITHM}

The algorithm of the method determines the train delay caused by voltage drops for a specified traffic intensity and feeding technology (such as: AT, BT, AT + HV, or BT + HV). In this paper intensity is defined as the periodicity of trains leaving a railway station for another.
A flowchart of the algorithm can be found in Figure 2, and a written description will follow.

1. Time $_{0}=0$, Position $_{0}=0$

2. Let out a train on the railway track if it is time

3. Load flow calculations

4. Outputs: $U, \theta, P_{G}$ (frequency converters), $P_{D}$, Velocity and $Q_{D}$ (Rc-locomotives).

5. Position $_{t+1}=$ Position $_{t}+$ Velocity $t \cdot \Delta$ Time, Time $_{t+1}=$ Time $_{t}+\Delta$ Time

6. Remove trains that have reached their destination

7. Was the recently removed train injected after the first train had been removed? If "Yes", move to the next step, if "No", move up to point 2

8. Algorithm halts. Throughput time of the train is displayed, detailed data are stored.

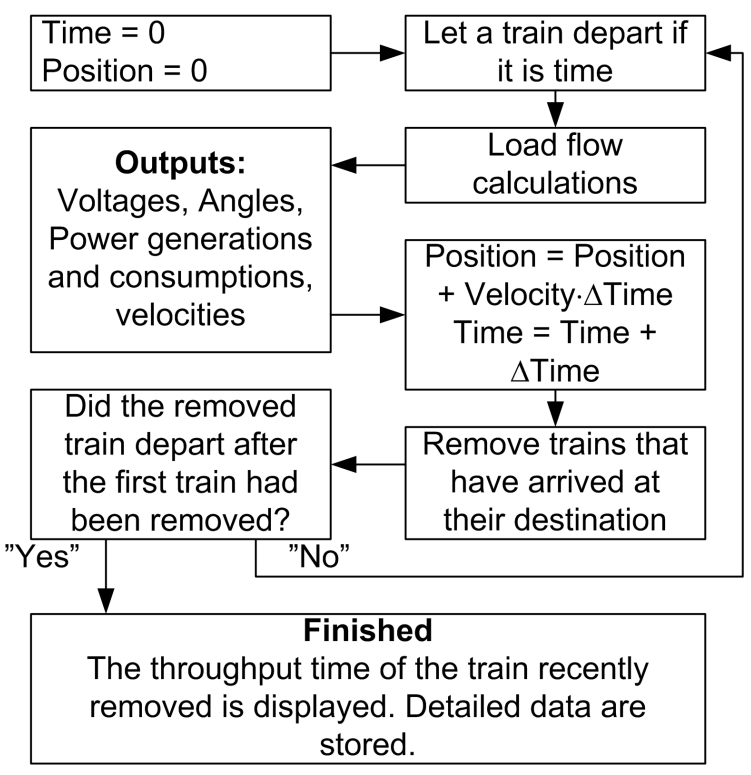

\section{Figure 2 A schematic flowchart describing the overall idea of the algorithm.}

Some exceptions from the ordinary way of doing power flow calculations will be brought up. These exceptions are related to the frequency converters as well as to the locomotives.

Normally, when using Newton Raphson for power flow calculations, $P_{G}, P_{D}, Q_{G}$, and $Q_{D}$ are treated as constants. Here, however, some of these might be functions of $U$ or $\theta$ with nonzero partial derivatives. This needs to be considered when determining the Jacobian matrix of the iteration.

The frequency converters are no ordinary PU nodes, because the power production $P_{G, k}$ is not fixed but dependent on the demands of the locomotives. The partial derivative of $P_{G, k}$ with respect to $U_{m}$ and $\theta_{m}$ can be found described thoroughly in [7]. Note that [5], [6] does not treat $Q_{G}$ (which is a part of equation 7) as $U$ or $\theta$ dependent at all. As the iteration proceeds, $P_{G, k}$ is updated as $P_{G, k, t+1}=P_{k, t}^{n e t}$ instead of being constant as in a normal PU-node. 
Nor are the locomotives ordinary PQ nodes. $P_{D}$ is as described in equation 4 modeled as an affine function of the voltage. Therefore the partial derivatives of $P_{D}$ are quite straightforward. The $Q_{D}$-derivatives are lengthier, but indeed derivable.

For a set of traffic intensities with known probabilities of occurrence, the algorithm may be run for each scenario. Thereafter weighting each simulated delay time together gives an expected time of delay. This expectation value may be treated as a cost that can be compared to the costs of investments in stronger feeding systems.

\section{THE TEST SYSTEM}

\section{The test system topology}

The simulations were performed on a three city test system. The test system has four different configurations, looking as illustrated in Figure 3. One can see that in the AT case, the initial impedance of the catenary earlier described gives rise to an extra node. In Figure 3: the dark gray square symbolizes a train; the light gray circles symbolizes cities where the converters are situated; the left hand side transformers take the voltage up to catenary level from the converters, while the right hand side transformers take the voltage up another level to the HV line. Distances between the cities are set to be $60 \mathrm{~km}$, and the converter-city distances as well as the catenary-transmission line distances are assumed to be negligible.
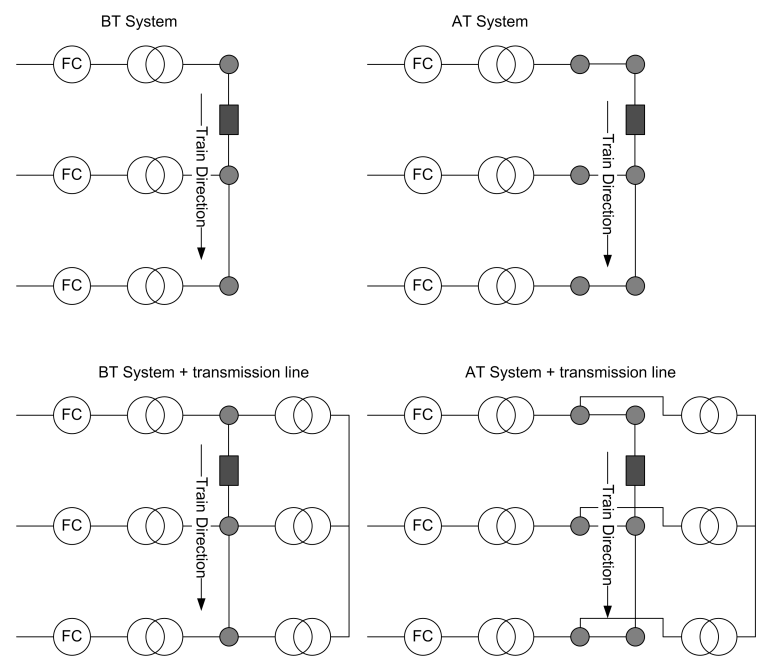

Figure 3 An illustration of the test system simulated. Four kinds of feeding technologies were investigated.

Catenaries, Transformers, and The Transmission Line The BT lines of the test system are of the $1092 \AA$ type [1], [2]. The AT impedances used in this study, are of type 120 2AT Fö [2]. This modeling is supported by [8].

HV line impedance data used in this model were retrieved from [1]. The figures of [2] are of approximately the same size as those of [1]. In this paper, the length of the lowvoltage part of the HV line (which is a piece of the catenarytransmission line distance) is assumed to be negligible.

There seem to be mainly two models of $\frac{16.5}{132} \mathrm{kV}$ transformers used. One model that is specified for $16 \mathrm{MVA}$, and another that is specified for 25 MVA [1]. Normally, the lower rated transformers are connecting the HV line and catenary further away from the feeding points, while the higher rated ones are connecting the lines in the vicinity of the feeding points. This is illustrated schematically in Figure 1. In the test system no 16 MVA transformers are used.

\section{Frequency Converters}

Since the static converters used today are set to act as rotating converters with the same phase angle on the $50 \mathrm{~Hz}$ side, in the simulations done, the $50 \mathrm{~Hz}$ side phase angles are all set to be zero.

Realistic values of the $50 \mathrm{~Hz}$ net parameters $X_{50}$ and $Q_{50}$ can be found in [6], while values of $U^{g}, U^{m}, X_{q}^{g}, X_{q}^{m}$, and transformer impedances can be found listed in [7] and [11]. Also the physical limitations of the rotary converters are listed in [7] and [11], where the latter contains more details. In the test system, the converter type Q38/Q39 is assumed. To be specific, the data of the converters located in Österås found in [6] was used.

\section{NUMERICAL EXAMPLE}

Curves of the maximal continuous power consumption of a $\mathrm{Rc}$ locomotive for five different voltages, as well as the running-resistance power when assuming flat ground and goods trains consisting of only one train and 30 wagons weighting 1600 metric tons, can be found plotted against velocity in Figure 4. These curves are extracted by measuring forcevelocity plots at certain points, recalculating them to powervelocity plots, and thereafter estimating them numerically to polynomials. The power consumption plots suited best for sixth order polynomials, while the resistive power plot were best modeled as a second order polynomial.

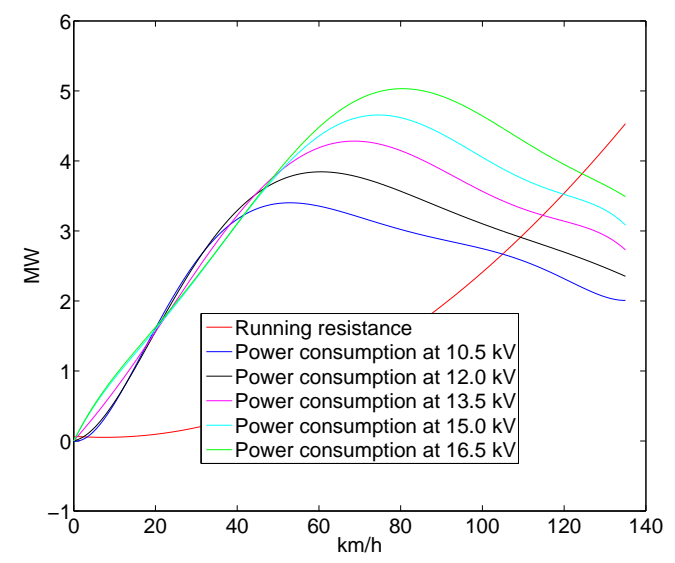

Figure 4 The increasing function represents the running-resistance-power. The remaining five functions illustrate the maximal power consumption of a typical Rc locomotive for certain pantograph voltages. All the plots represent the power dependency (MW) of velocity $(\mathbf{k m} / \mathbf{h})$.

The parameters $a, b, c$, and $d$ of equation 8 were by least squares determined to be $a=0.1956, b=0.5875, c=$ 3.3147 , and $d=69.8560$. 


\begin{tabular}{|c|c|ccc|}
\hline AT & HV & TP=5 & TP=10 & TP=20 \\
\hline Yes & Yes & 60 & 58.9 & 58.4 \\
Yes & No & 60.1 & 58.9 & 58.4 \\
No & Yes & 62.2 & 60.1 & 59.2 \\
No & No & 62.8 & 60.2 & 59.3 \\
\hline
\end{tabular}

Table 1 Train throughput times for different feeding systems and different train departure periodicities. AT $=$ No, HV=No, means simply a BT fed system. AT $=$ Yes, HV=Yes means an AT system with supplementary high voltage line.

The time resolution chosen in the simulations, using the prior described algorithm, was 0.1 minutes. The simulation results, expressed as throughput times, for each train departure periodicity and feeding technology can be found in Table 1.

For the AT case, an expected delay can be calculated. For nominal voltage, i.e., $U=16.5 \mathrm{kV}$, the velocity would be $v=124.55 \mathrm{~km} / \mathrm{h}$ giving the throughput time 57.81 minutes. In the simplest case, assign probabilities $P=\frac{1}{3}$ for train periods 5,10 and 20 minutes. Then the expected delay can be calculated to be 1.29 minutes with HV line and 1.32 minutes without HV line. Similar calculations for BT fed trains give 2.69 minutes with HV line, and 2.96 minutes without any supplementary HV line.

\section{DISCUSSION}

The limitation of the model that is the most urgent to remedy is the neglecting of mass inertia of the trains. Another important improvement might be allowing a high power consumption of the locomotives for short periods of time, which is the case in real life.

In a further development of the model, it would be desirable to handle trains traveling in both directions, or even more irregularly. In such a situation, the definition of time table deviations might need to go down to an individual level regarding the trains.

\section{CONCLUSION}

A model has been compiled, and a simulation method has been developed treating train movements in time. A numerical example has been applied to a realistic test system, and results are briefly presented.

In this particular example, the voltage levels did not seem to be a dimensioning factor of the time table deviations. However, this might not be the case with other test system topology, or different kinds of train sets.

\section{ACKNOWLEDGEMENTS}

The authors would like to thank Anders Bülund and Thorsten Schütte for valuable information and encouraging discussions.

\section{REFERENCES}

[1] Banverket (2002), Power supply inquiry Östergötland / Småland - Appendix 18 (in Swedish), BK-dokument BKE 02/01.

[2] Banverket (2005), Impedances for KTL and $132 \mathrm{kV}, 30$ $k V$ and $15 k V M L$ (in Swedish), BK-dokument BKE $02 / 28$.

[3] Bülund A. (2004), KTH EME-seminary Railway Feeding Systems, 2004-03-26 (in Swedish), Anders Bülund PDF-slides

[4] Bülund A. (2006), Private oral conversation.

[5] Olofsson M., Andersson G., Söder L., Power Flow in a Public Grid Integrated Electric Traction System, 11th Power Systems Computation Conference, pp 209-215, Aug.-Sept., 1993

[6] Olofsson M. (1993), Power Flow Analysis of the Swedish Railway Electrical System, KTH.

[7] Olofsson M. (1996), Optimal Operation of the Swedish Railway Electrical System - An Application of Optimal Power Flow, KTH.

[8] Varju G. (1996), Simplified method for calculating the equivalent impedance of an AT system, Technical University of Budapest / Innotech Ltd.

[9] Östlund S. (2005), Electrical Traction (in Swedish), KTH.

[10] Östlund S. (2006), Private oral conversation.

[11] Banverket (2003), Description of different designs of mobile converters and technical data (in Swedish), BVH 543.16001 .

\section{AUTHOR'S ADDRESS}

Both of the authors can be contacted at

School of Electric Engineering

Electric Power Systems

Royal Institute of Technology (KTH)

Teknikringen 33

SE-100 44 Stockholm

Sweden

email: lars.abrahamsson@ee.kth.se lennart.soder@ee.kth.se 